EUV spectroscopy of

high-redshift $x$-ray objects

M. P. Kowalski, M. T. Wolff, K. S. Wood, T. W. Barbee, M. A. Barstow

June 16, 2010

SPIE Conference 7732 - 168

San Diego, CA, United States

June 27, 2010 through July 2, 2010 
This document was prepared as an account of work sponsored by an agency of the United States government. Neither the United States government nor Lawrence Livermore National Security, LLC, nor any of their employees makes any warranty, expressed or implied, or assumes any legal liability or responsibility for the accuracy, completeness, or usefulness of any information, apparatus, product, or process disclosed, or represents that its use would not infringe privately owned rights. Reference herein to any specific commercial product, process, or service by trade name, trademark, manufacturer, or otherwise does not necessarily constitute or imply its endorsement, recommendation, or favoring by the United States government or Lawrence Livermore National Security, LLC. The views and opinions of authors expressed herein do not necessarily state or reflect those of the United States government or Lawrence Livermore National Security, LLC, and shall not be used for advertising or product endorsement purposes. 


\title{
EUV spectroscopy of high-redshift x-ray objects
}

\author{
M. P. Kowalski ${ }^{* a}$, M. T. Wolff ${ }^{a}$, K. S. Wood ${ }^{a}$, T. W. Barbee, Jr., , M. A. Barstow ${ }^{c}$ \\ ${ }^{a}$ Naval Research Laboratory, Code 7655, Washington, DC, USA 20375; \\ ${ }^{b}$ Lawrence Livermore National Laboratory, MS L-353, 7000 East Ave., Livermore CA, USA 94550; \\ ${ }^{c}$ Dept. of Physics and Astronomy, University of Leicester, University Road, Leicester UK LE1 7RH
}

\begin{abstract}
As astronomical observations are pushed to cosmological distances $(z>3)$ the spectral energy distributions of X-ray objects, AGN for example, will be redshifted into the EUV waveband. Consequently, a wealth of critical spectral diagnostics, provided by, for example, the Fe L-shell complex and the O VII/VIII lines, will be lost to future planned Xray missions (e.g., $I X O, G e n-X$ ) if operated at traditional X-ray energies. This opens up a critical gap in performance located at short EUV wavelengths, where critical X-ray spectral transitions occur in high-Z objects. However, normalincidence multilayer-grating technology, which performs best precisely at such wavelengths, together with advanced nanolaminate replication techniques have been developed and are now mature to the point where advanced EUV instrument designs with performance complementary to $I X O$ and Gen- $X$ are practical. Such EUV instruments could be flown either independently or as secondary instruments on these X-ray missions. We present here a critical examination of the limits placed on extragalactic EUV measurements by ISM absorption, the range where high-z measurements are practical, and the requirements this imposes on next-generation instrument designs. We conclude with a discussion of a breakthrough technology, nanolaminate replication, which enables such instruments.
\end{abstract}

Keywords: Extreme Ultraviolet, High-Resolution Spectroscopy, Diffraction gratings, Multilayer coatings, Nanolaminate replication, High-redshift, AGN

\section{INTRODUCTION: SUMMARY OF THE PROBLEM AND THE PROPOSED SOLUTION}

Much of the emphasis in NASA's current Strategic Plans is on understanding the early universe and how it has evolved to its present state ${ }^{1}$. As astronomical observations are pushed to cosmological distances $(z>3)$ the spectral energy distributions and important lines of X-ray objects, AGN for example, will be redshifted into the EUV waveband ( 100$1000 \AA / 0.1-0.01 \mathrm{keV})$, including for example, the Fe L-shell complex $(\sim 60-6 \AA / 0.2-2.0 \mathrm{keV})$ and the O VII/VIII lines $(\sim 20 \AA / 0.5 \mathrm{keV})$. (An analogous situation exists for JWST relative to HST.) We illustrate this effect at a redshift (z) of 5 for the strong O VII and VIII lines in Figure 1, which shows the Newton-XMM RGS spectrum of the prototypical Seyfert galaxy NGC 1068 taken from Kinkhabwala et $\mathrm{al}^{2}$. A wealth of critical spectral diagnostics may be lost if future planned $\mathrm{X}$-ray missions are optimized for traditional energies. For example, public documents put the low energy limit for $I X O^{3}$ at $0.3 \mathrm{keV}(\sim 41 \AA)$, and while the limit for Gen- $X^{4}$ is currently $0.1 \mathrm{keV}(\sim 123 \AA)$, the EUV response might be traded in future design refinements when practical matters such as detector size and budget limitations force tough choices.

This potential critical gap in performance is located at short EUV wavelengths, where the critical X-ray spectral transitions occur in high-z objects. Fortunately, normal-incidence multilayer-grating technology performs best precisely at such wavelengths, and we propose it as a solution to this problem. Multilayer gratings have a significant advantage in the achievement of both high resolving power $(\mathrm{R}=\lambda / \Delta \lambda)$ and high effective area in one instrument, but at the disadvantage of a limited bandwidth. However, the latter may be overcome by designing wavebands to match the location of spectral lines, and then building a suite of spectrometers. In Figure 2, we show a SMEX concept design, possible with present technology, whose predicted performance is shown in Figure 1. Peak R and effective area lie in the ranges 8640-11400 and 15-60 $\mathrm{cm}^{2}$, respectively. Where responses overlap, the effective area at the wavelengths of important spectral lines exceeds that of both Chandra and EUVE by an order of magnitude, and the resolving power is better by factors of 5 and 30, respectively. Most importantly, the multilayer designs can be tailored to locate the peak response of any spectrometer to any wavelength. However, this SMEX design was optimized for observation of galactic targets.

\footnotetext{
michael.kowalski@nrl.navy.mil; phone 1202 767-2781; fax 1202 767-0497
} 
In the following sections we discuss important issues for extragalactic observations including absorption by the galactic interstellar medium (ISM), the flux of distant X-ray objects, and practical instrument requirements (effective area, wavelength coverage, resolving power). We conclude with a discussion of a breakthrough technology, nanolaminate replication, which enables such instruments ${ }^{5-9}$.

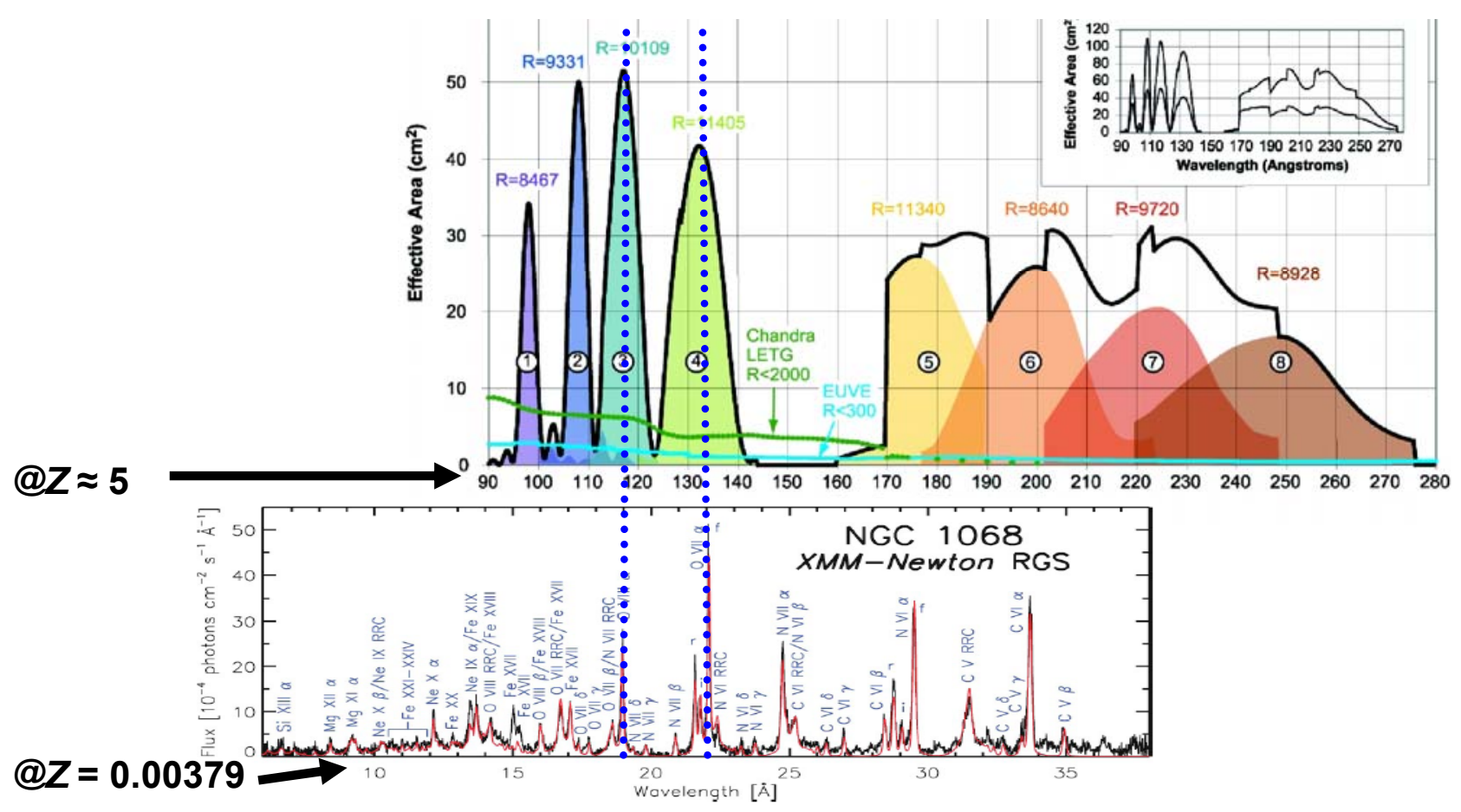

Figure 1. (bottom) XMM-Newton RGS X-ray spectrum ${ }^{2}$ of prototypical Seyfert 2 galaxy NGC 1068 ( $\left.\mathrm{z}=0.00379\right)$. (top) Effective area and resolving power (R) for a SMEX concept design (Figure 2) for a suite of 8 highresolution multilayer-grating EUV spectrometers. The abscissae of the two figures have been offset to illustrate the scaling by a factor of $(1+z)$, where the O VII lines fall at the peaks of two of these EUV spectrometers when the galaxy is shifted to $\mathrm{z}=5$.

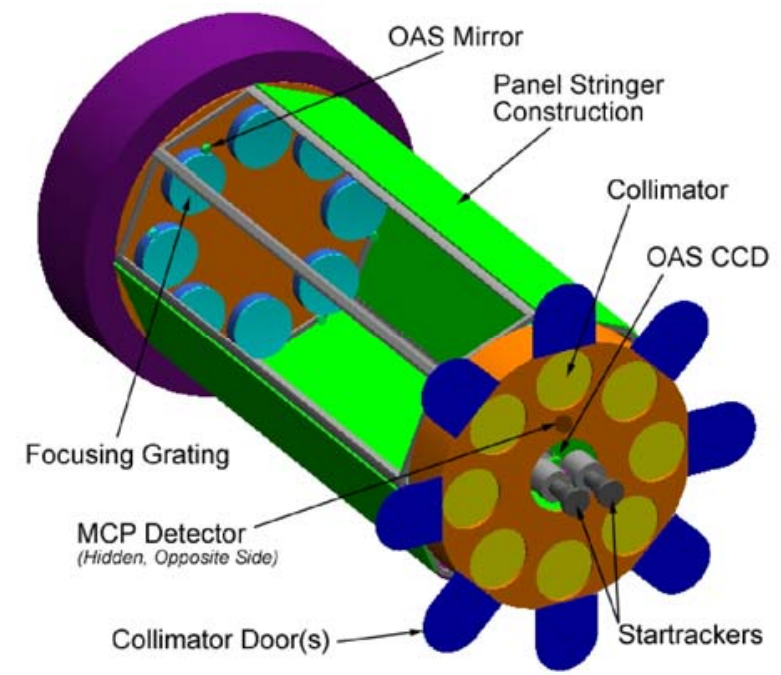

Figure 2. SMEX Concept Design for a Suite of 8 high-resolution multilayer-coated EUV spectrometers ${ }^{10}$. 


\section{GALACTIC ISM TRANSPARENCY}

Prior to the 1970s, it was generally believed that EUV astronomy was impossible. This assertion was based on the knowledge that most elements have electron binding energies in the range $10-100 \mathrm{eV}$ and on calculations ${ }^{11}$ that assumed an average interstellar medium (ISM) density for hydrogen of $1 \mathrm{~cm}^{-3}$, which was inferred from the available low spatial resolution data produced by $21-\mathrm{cm}$ radio surveys. However, Far-UV satellite measurements have showed that the local density within a few hundred parsec was much less than the average integrated over longer path lengths, thus allowing diverse galactic studies at EUV wavelengths. The total galactic column density remains important for extragalactic observations. Radio surveys of higher angular resolution reveal a general decrease in neutral hydrogen column density $\left(n_{\mathrm{H}}\right)$ with increasing galactic latitude ${ }^{12-13}$, the average value being proportional to $2.9 \times 10^{20} \mathrm{csc}|\mathrm{b}| \mathrm{cm}^{-2}$. The structure is patchy, with a large dispersion $\left(1.4 \times 10^{20} \mathrm{~cm}^{-2}\right)$ in the average. This patchiness contains holes that allow extragalactic EUV observations. The lowest column density of $4.4 \times 10^{19} \mathrm{~cm}^{-2}$ is found in the 'Lockman hole' $\left(\sim 15 \mathrm{deg}^{2}\right)$, and Figure 3 shows the histogram of column densities taken from Dickey and Lockman ${ }^{13}$. When Figure 3 is normalized to $4 \pi$ steradian, there are $300 \mathrm{deg}^{2}$ of sky with $n_{\mathrm{H}}<1.0 \times 10^{20} \mathrm{~cm}^{-2}$, which is comparable to the XMM-Newton AGN X-ray survey ${ }^{14}$ and $1300 \mathrm{deg}^{2}$ with column densities less than $1.6 \times 10^{20} \mathrm{~cm}^{-2}$. The Chandra and HST deep sky surveys are $\sim 1$ $\mathrm{deg}^{2}$ and are located in regions of low neutral hydrogen column density. Thus we conclude that sufficient solid angle is available for extragalactic EUV observations of high-z AGN. We also note that approximately 1100 EUV sources were cataloged in the EUVE and the ROSAT WFC surveys where exposures were only $5 \times 10^{3} \mathrm{~cm}^{2}$-sec. About 20 of these are low-z AGN spanning all classes (Seyfert, BL Lac, QSO) ${ }^{15}$.

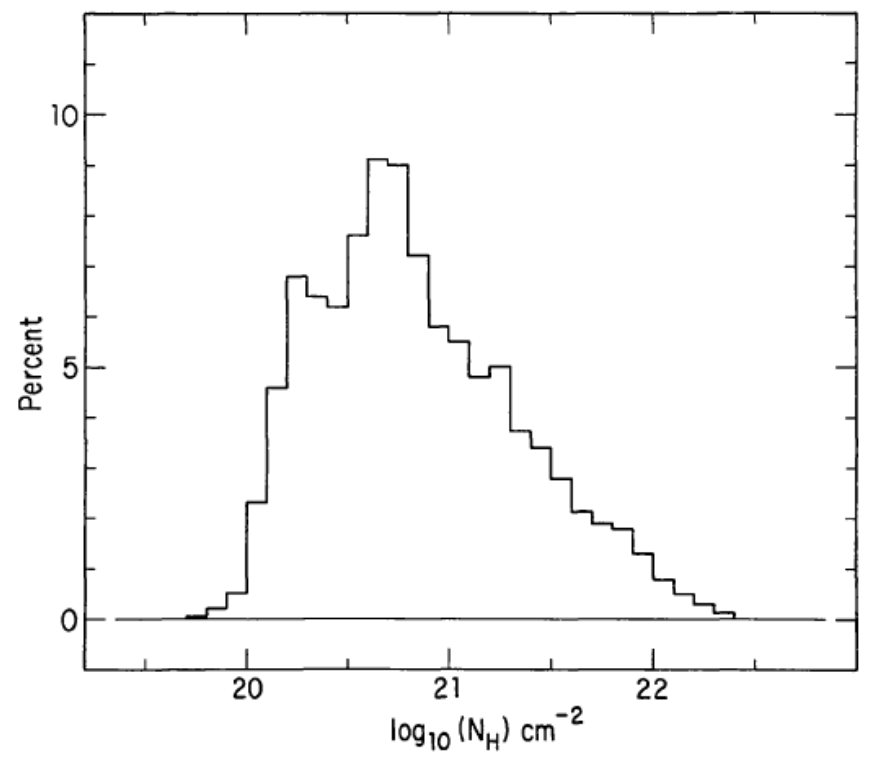

Figure 3. Histogram of the fraction of the sky as a function of total integrated column density of neutral hydrogen, taken from Dickey and Lockman ${ }^{13}$.

The transparency of the galactic ISM as a function of wavelength is a second critical parameter in determining the feasibility of extragalactic EUV observations. The total ISM absorption cross section is dominated by H I, He I, He II, and $\mathrm{H}_{2}$, with metals accounting for less than $10 \%$ of the total in the EUV. In Figure 4 we show the cross section as a function of wavelength taken from the model of Rumph, Bowyer, and Vennes ${ }^{16}$, which is an update of Cruddace et $\mathrm{al}^{17}$. We have used the results in Figure 4 to calculate the transmission for different assumed values of $n_{\mathrm{H}}$, and in Figure 5 we plot the inverse of the transmission (Tr) as a function of wavelength. This quantity may be regarded as the required factor increase in observation time for a given signal-to-noise ratio. For targets in the Lockman hole this factor ranges from $10-100$ at wavelengths up to $140 \AA$. Choosing a factor of $1000(0.1 \%$ transmission) limits observations to $<130 \AA$ for $n_{\mathrm{H}}=1.0 \times 10^{20} \mathrm{~cm}^{-2}$ and $<110 \AA$ for $n_{\mathrm{H}}=1.6 \times 10^{20} \mathrm{~cm}^{-2}$. 

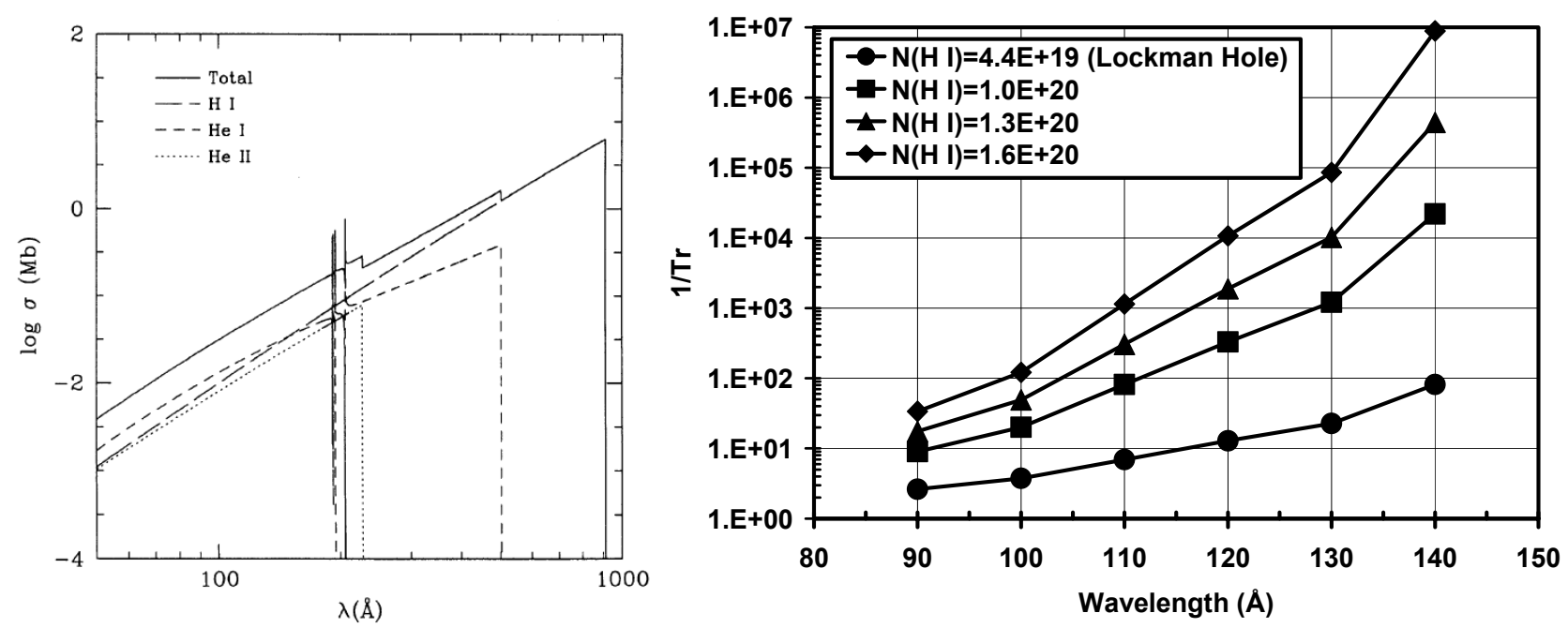

Figure 4 (left). ISM absorption cross section, taken from Rumph, Bowyer, and Vennes ${ }^{16}$.

Figure 5 (right). Inverse of ISM transmission as a function of wavelength for different assumed values of $n_{\mathrm{H}}$.

\section{EXTRAGALACTIC AGN FLUX: AN EXAMPLE WITH O VII}

Independent of intrinsic evolution, the flux of any astronomical source is reduced considerably at cosmological distances. As a representative case we consider the flux in the O VII He-like triplet in NGC 1068. We have chosen this line because it is one of several that are important ${ }^{18}$ in understanding the WHIM (Warm-Hot Intergalactic Medium). The measured fluxes ${ }^{2}$ at $z=0.00379$ are $9.59,1.75$, and $4.50 \times 10^{-4}$ photons $\mathrm{cm}^{-2} \mathrm{sec}^{-1}$ in the $\mathrm{f}(22.101 \AA), \mathrm{i}(21.803 \AA)$, and $\mathrm{r}$ $(21.602 \AA)$ lines, respectively. We note that a similar flux is found for the f-line in Mkn 3 at $z=0.013509$, after adjusting for distance ${ }^{19}$, and other AGN might have intrinsically brighter lines. It is not appropriate to scale observed fluxes at cosmological distances using only the inverse square law. We have used the following equations, which are derived from Sandage ${ }^{20}$ Eq. (19) for luminosity, evaluated for a closed universe $(K=0)$ to scale the flux to $z=5$. The integrated line luminosity is given by:

$$
\mathrm{L}=4 \pi \mathrm{c}^{2} \mathrm{H}_{\mathrm{o}}^{-2}(1 / 2)^{-4}(1 / 2)^{2}\left\{\mathrm{z}-\left[(1+\mathrm{z})^{1 / 2}-1\right]\right\}^{2} \mathrm{x} \text { flux, }
$$

and thus to scale the f-line flux to $z=5$ :

$$
\text { f-line flux }=9.59 \times 10^{-4} \times\left\{0.00379-\left[(1+0.00379)^{1 / 2}-1\right]\right\}^{2} /\left\{5-\left[(1+5)^{1 / 2}-1\right]\right\}^{2}=2.74 \times 10^{-10} \text { photons cm } \mathrm{sec}^{-1} .
$$

From the previous section, absorption from the galactic ISM would reduce this flux further by greater than an order of magnitude in the Lockman hole at wavelengths less than $\sim 140 \AA$. However, X-ray luminosity is highly correlated with the cosmic star formation rate, which was a factor of 10-100x higher at $\mathrm{z}=1-3$ than at present ${ }^{4}$. Thus we assume that luminosity evolution and ISM absorption approximately cancel each other, and we use the calculated flux in Eq. (2) to set instrument requirements.

\section{INSTRUMENT REQUIREMENTS}

We should emphasize that the calculation done in section 3 is part of a preliminary attempt to understand requirements, but is in no way a flow-down from a mission-driver. The resulting scale shown below, an EUV instrument comparable to or complementary to Gen-X, poses scientific questions far beyond the starting premise, because it would constitute a breathtaking leap for all EUV astronomy (including, e.g., Galactic investigations of white dwarfs, ISM, stellar coronae, nearby neutron starts including millisecond pulsars - central issues of stellar evolution in our vicinity). A companion paper in these proceedings ${ }^{21}$ describes much more modest near-term missions for that other EUV science; those missions would be part of the preparatory effort for reaching the major facility under discussion here, just as Einstein and Chandra have brought us to where a Gen- $X$ mission can be studied. Exploring the full potential of that large EUV facility architecture is far beyond the scope of this paper. Also beyond scope is architecture optimization to enable a 
prioritized set of Galactic and extragalactic science goals. However, what is in scope is to emphasize that crucial technology needed to realize this major development is far along in development, in fact surprisingly advanced, and ready to be evaluated as a competitive candidate for the central technology of a major new facility. It is this technology breakthrough in nanolaminate replication (section 5) that makes the following discussion more than just an exercise in the redshifting of spectra and calculation of photon yields.

\subsection{Effective Area}

We assume an integration time of $10^{6} \mathrm{sec}$, which is comparable to that used for pointed observations with the Fermi and Compton GRO satellites. Thus $10^{6} \mathrm{~cm}^{2}\left(100 \mathrm{~m}^{2}\right)$ of effective area are needed to produce 274 counts in the O VII $\mathrm{f}$-line and $\sim 50$ counts in the weakest triplet i-line. These are sufficient statistics to calculate standard plasma diagnostic ratios with acceptable precision. The required effective area is comparable designed for Gen-X. However, it should be noted that the grazing-incidence design of Gen-X requires that $\sim 10^{4} \mathrm{~m}^{2}$ of optical surface must be fabricated to achieve that value of effective area ${ }^{4}(\sim 1 \%$ ratio), while normal-incidence multilayer-optics designs are overall more efficient $(\sim 5 \%$ ratio) at EUV wavelengths.

\subsection{Wavelength Coverage}

At $z=5$, the wavelength of the O VII He-like triplet f-line is $132.6 \AA$. The FWHM of the effective area profile in Figure 1 (driven by the multilayer response) is $10 \AA$, and this produces a useful redshift range of $z=4.77-5.23$ for the observation parameters discussed above. However, multilayer designs can be easily tailored to broaden the FWHM by approximately a factor of 2 at a cost of a factor of $1 / 2$ in the peak effective area (the area under the multilayer wavelength response curve is approximately conserved). Thus, three spectrometers might be designed whose combined effective area would span the range 100-140 $\AA$ without gaps, similar to that of the four longest wavelength spectrometers in Figure 1 for the range 170-275 $\AA$. The wavelength range 100-140 $\AA$ corresponds to a redshift range $z=3.5-5.3$ for the $O$ VII f-line. An added benefit is that X-ray transitions at wavelengths longer than the O VII lines would also be observable in such an instrument but at lower redshift (e.g., $\mathrm{C}_{\mathrm{K}}$-edge: $z \sim 2$ ).

\subsection{Resolving Power}

A spectral resolving power of only R 130 will separate components of the O VII He-like triplet at $z=5$, which presents no significant challenge even to present technology. However, much higher resolving power is required to separate AGN lines from those of the galactic ISM, which may be seen in absorption for those AGN with strong continua (e.g., 3C273), as well as multiple components from intergalactic gas at varying redshift along the line-of-sight. Thus it is prudent to use the highest $\mathrm{R}$ practical to avoid confusion in identifying spectral components. We have already achieved $\mathrm{R}=4000$ with a multilayer grating instrument (section 5) of 2-m focal length (f22). For $\mathrm{R} \sim 2000$ and a comparable focal length ratio, the focal distance would be comparable to that required by Gen-X $(\sim 100 \mathrm{~m})$.

\section{TECHNOLOGY}

For over two decades we at the Naval Research Laboratory (NRL) in collaboration with the Lawrence Livermore National Laboratory (LLNL) have pursued the goal of achieving simultaneously high effective area and high resolution in a normal-incidence EUV spectrometer by using ultra-smooth holographic ion-etched gratings that have been coated with high-reflectance multilayers. In a companion paper in these proceedings ${ }^{21}$ we review the design and operation of multilayer coatings and summarize our success in their application to gratings. This work has culminated in the J-PEX high-resolution EUV spectrometer, which has flown successfully on two NASA sounding rocket missions. J-PEX has the largest EUV multilayer gratings yet fabricated, which are $16 \times 9 \mathrm{~cm}$ in size and have a fused silica substrate. However, to ensure that figure is maintained, grating designers keep the ratio of substrate thickness to diameter constant as optics get larger. Thus, without weight relief areal density grows linearly with radius and total weight grows as the cube of the radius. The areal density of the $J-P E X$ gratings is $\sim 3 \mathrm{~kg} \mathrm{~m}^{-2}$, but is already $\sim 10 \mathrm{~kg} \mathrm{~m}^{-2}$ for gratings designed for the SMEX mission describe in section 1. Even with weight relief total weight and cost rapidly grows beyond practical limits when considering large next-generation instruments such as that described here.

One solution to this problem is called the Actuated Hybrid Mirror Concept ${ }^{22-24}$, where separate approaches are used to realize mirror finish and figure (Figure 6). Finish is provided by a breakthrough technology. Nanolaminates are the result of the extension of short wavelength reflecting multilayer coating technology ${ }^{25-27}$ to the breadth of the Periodic Chart in composition and a range of atomic arrangements spanning non-crystalline/amorphous to single crystal. 
Nanolaminates with thickness up to 400 microns have been synthesized for both scientific and technological investigations. Thus, a replicating nanolaminate may be a single layer or for our purposes an EUV multilayer structure backed by a macroscopic nanolaminate. The capability to make large-area nanolaminate structures without degradation from unwanted flaw sources is a technical tour de force.

To accommodate figure, a nanolaminate facesheet with the required finish (and hence reflectance) is replicated off a precision mandrel and then bonded to a substrate. The substrate is rib-stiffened $\mathrm{SiC}$, cast to near net shape and generated to the required optical figure. Surface parallel actuators embedded in the substrate ribs are used to actively control mirror figure. Finally, a sensing system may be used to determine real time mirror figure and provide control-inputs to the actuators. This concept has established the ability to fabricate $1.2 \mathrm{~m}$ mirrors (Figure 7), where the nanolaminate facesheets were developed and applied by Dr. Barbee (LLNL). He has replicated single- and multilayer-foil mirrors of a variety of material combinations ${ }^{28}$ from precision masters, which have high strength (free standing), atomic smoothness, spherical figure, 1-m diameters, and thicknesses from two monolayers $(\sim 4 \AA)$ to hundreds of monolayers $(>5000 \AA)$. Multiple copies have been replicated from a single master with no degradation in roughness in the master or the copies. The nanolaminate foil areal density is $<0.5 \mathrm{~kg} \mathrm{~m}^{-2}$ before electromagnetic components are mounted, and a target of $<2 \mathrm{~kg}$ $\mathrm{m}^{-2}$ with control elements. Diffraction-limited resolution has been obtained. The next step is an obvious one, namely to extend nanolaminate replication technology to high-quality gratings, and thus create the capability to produce large, efficient grating collecting area economically for high-resolution normal-incidence EUV spectroscopy.

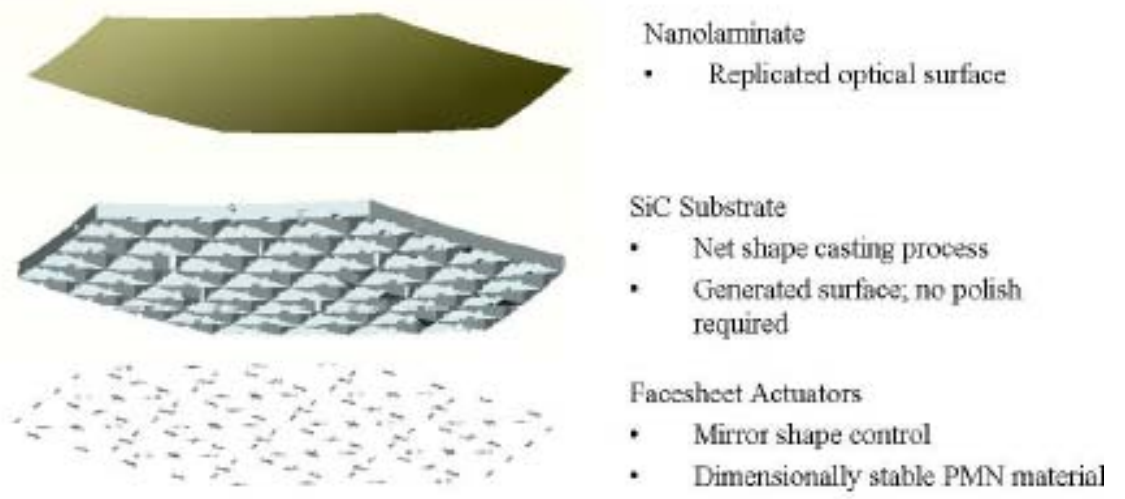

Figure 6. Actuated Hybrid Mirror (AHM) Concept ${ }^{16}$.

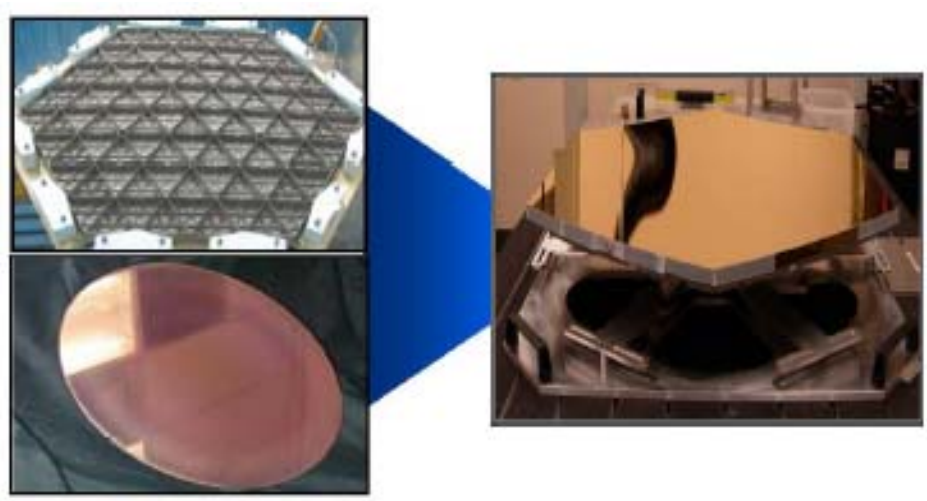

Figure 7. Demonstration of the AHM Concept ${ }^{16}$.

\section{CONCLUSIONS}

The goal of understanding the early Universe presents a great challenge as it largely requires observation of distant objects, where fluxes received at Earth are generally small at most wavelengths and whose spectra are redshifted from 
the source rest frame. A particular difficulty exists at the low-energy end of the X-ray spectrum where important transitions (e.g., Fe, O) will be redshifted to the EUV. We have examined important issues for extragalactic EUV spectroscopy including absorption by the galactic ISM and the flux of distant X-ray objects, and we have calculated practical instrument requirements (effective area, wavelength coverage, resolving power). We find that observations of important spectral lines in high-z AGN are practical, assuming effective areas of order $10^{6} \mathrm{~cm}$ in $10^{6}$ sec and with resolving powers sufficient to disentangle source components from those caused by the galactic ISM and by intervening intergalactic gas. Without such capability a gap in coverage will exist precisely at the wavelengths of critical spectral transitions in high-z AGN. As currently designed Gen- $X$ may cover this gap, but $I X O$ will not. It seems prudent to seek alternatives.

While multilayer-grating technology is sufficiently mature for the purposes of galactic high-resolution EUV spectroscopy, the large collecting areas required to observe distant extragalactic sources profitably requires new developments. Fortunately, at least one candidate technology exists. Nanolaminate replication, a breakthrough technology that is derived from multilayer fabrication techniques, has made rapid progress, and high-quality meter-class mirrors have been fabricated. We advocate extending this technology to the fabrication of high-resolution gratings to provide the required large collecting area economically in both weight and cost.

\section{ACKNOWLEDGEMENTS}

The research at the Naval Research Laboratory was supported by the Office of Naval Research under W.U. 3641: Application of Multilayer Coated Optics to Remote Sensing and by NASA-Defense Purchase Request S-47440F: A Study of the Physics and Composition of White Dwarf Atmospheres using High Resolution EUV Spectroscopy. Part of the research was done at the National Synchrotron Light Source, which is sponsored by the U.S. Department of Energy under contract DEAC02-76CH00016. Prepared by LLNL under Contract DE-AC52-07NA27344.

\section{REFERENCES}

[1] science.nasa.gov/about-us/science-strategy/

[2] Kinkhabwala, A., Sako, M., Behar, E., Kahn, S. M., Paerels, F., Brinkman, A. C., Kaastra, J. S., Gu, M. F. and Liedahl, D. A., "XMM-Newton Reflection Grating Spectrometer Observations of Discrete Soft X-ray Emission Features from NGC 1068," Ap. J. 575, 732-746 (2002).

[3] ixo.gsfc.nasa.gov/technology/xgs.html

[4] Windhost, R., Cameron, R. A., Brissenden, R. J., Elvis, M. S., Fabbiano, G., Gorenstein, P., Reid, P. B., Schwartz, D. A., Bautz, M. W., Figueroa-Feliciano, E., Petre, R., White, N. E. and Zhang, W. W., "Generation-X: An X-ray observatory designed to observe first light objects," New Ast. Rev. 50, 121-126 (2006).

[5] Barbee, Jr., T. W., "Multilayer Structures: Atomic Engineering in its Infancy", [Physics, Fabrication and Applications of Multilayered Structures], Plenum Press, New York, 17 (1988).

[6] Barbee, Jr., T. W. and Keith, D. L., "Synthesis of Metastable Materials by Sputter Deposition Techniques," J. Metals 32, 11 (1980).

[7] Barbee, Jr., T. W., "Nano-Laminates: A New Class of Materials for Aerospace Applications", Proc. IEEE Aero. Conf. 4, 1745-1754 (2003).

[8] Ealey, M. A., "Large Optics in the 21st Century: A Transition from Discrete Manufacturing to Highly Integrated Techniques", Proc. IEEE Aero. Conf. 4, 1705-1716 (2003).

[9] Redding, D. C. Shi, F., Basinger, S. A., Cohen, D., Green, J. J., Lowman, A. E. and Ohara, C. M., "Wavefront Control for Large Space Optics," Proc. IEEE Aero. Conf. 4, 1729-1744 (2003).

[10] Kowalski, M. P., Cruddace, R. G., Wood, K. S., Yentis, D. J., Wolff, M. T., Laming, J. M., Gursky, H., Carruthers, G. R., Barbee, Jr., T. W., Kordas, J. F., Mauche, C. W., Fritz, G. G., Varlese, S. J., Barstow, M. A., Fraser, G. W., Siegmund, O. H. W., Welsh, B. Y., Brickhouse, N. S., Dupree, A. K., Brown, A., Bruhweiler, F. C., Cameron, A. C., Holberg, J. B., Howell, S. B., Jordan, C., Linsky, J. L., Matthews, S. A., Sion, E. M. and Werner, K., "Proposed Mission Concept for the Astrophysical Plasmadynamic Explorer (APEX): An EUV High Resolution Spectrscopic SMEX," Proc. SPIE 5164, 1-16 (2003).

[11] Aller, L. H., "Some aspects of ultraviolet satellite spectroscopy," Pub. Ast. Soc. Pac. 71, 324-329 (1959).

[12] antwrp.gsfc.nasa.gov/apod/image/0101/nHI_alt skyview big.gif

[13] Dickey, J. M. and Lockman, F. J., "H I in the Galaxy," Ann. Rev. Ast. Astrophys. 28, 215-261 (1990). 
[14] Mateos, S., Warwick, R. S., Carrera, F. J., Stewart, G. C., Ebrero, J., Ceca, R. D., Caccianiga, A., Gilli, R., Page, M. J., Treister, E., Tedds, J. A., Watson, M. G., Lamer, G., Saxton, R. D., Brunner, H. and Page, C. G., "High precision X-ray $\log N-\log S$ distributions : implications for the obscured AGN population," Ast. Astrophys. 492, 51-69 (2008).

[15] Fruscione, A., [Astrophysics in the Extreme Ultraviolet], Kluwer, Dordrecht, 381 (1996).

[16] Rumph, T., Bowyer, S. and Vennes, S, "Interstellar Medium Continuum, Autoionization, and Line Absorption in the Extreme Ultraviolet," Ast. J. 107, 2108-2114 (1994).

[17] Cruddace, R. G., Paresce, F., Bowyer, S. and Lampton, M., "On the opacity of the interstellar medium to ultrasoft x-rays and extreme-ultraviolet radiation," Ap. J. 187, 497-504 (1974).

[18] Paerels, F. B. S. and Kahn, S. M., "High-Resolution X-ray Spectroscopy with Chandra and XMM-Newton," Ann. Rev. Ast. Astrophys. 41, 291-342 (2003).

[19] Sako, M., Kahn, S. M., Paerels, F. and Liedahl, D. A., "The Chandra High-Energy Transmission Grating Observation of an X-ray Ionization Cone in Markarian 3,” Ap. J. Let. 543, L115-L118 (2000).

[20] Sandage, A. [Galaxies and the Universe], The University of Chicago Press, Chicago, 778 (1975).

[21] Kowalski, M. P., Wood, K. S., Barstow, M. A. and Cruddace, R. G., "It's time for a new EUV orbital mission," Proc. SPIE 7732, this volume (2010).

[22] MacEwen, H. A., "Separation of functions as an approach to development of large space telescope mirrors," 2004, Proc. SPIE 5166, 39-48 (2004).

[23] www8.nationalacademies.org/astro2010/publicview.aspx

[24] Hickey, G., Barbee, Jr., T. W., Ealey, M. and Redding, D.C., “Acuated Hybrid Mirrors for Space Telescopes," Proc. SPIE 7731, this conference (2010).

[25] Barbee, Jr., T. W., "Sputtered layered synthetic microstructure (LSM) dispersion elements," Proceedings of the Topical Conference on Low Energy X-Ray Diagnostic (Monterey, CA) p. 131 (1981).

[26] Barbee, Jr., T. W., [Synthetic Modulated Structures], Academic Press, New York, 313 (1985).

[27] Barbee, Jr., T. W., "Multilayers for X-Ray Optics," Opt. Eng. 25, 898-915 (1986).

[28] Hickey, G. S., Lih, S.-S. and Barbee, Jr., T. W., "Development of Nanolaminate Thin Shell Mirrors," Proc. SPIE 4849, 63-76 (2003). 